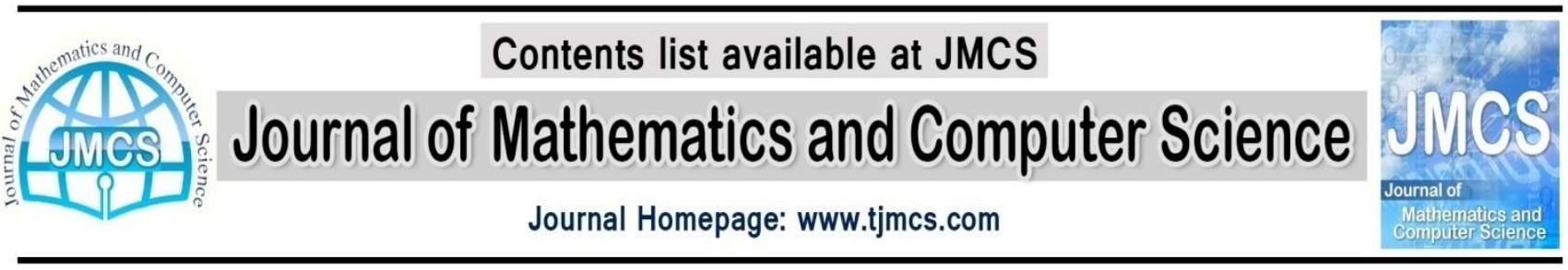

\title{
Integrated High Accuracy Multiquadric Quasi-interpolation Scheme for Solving the Nonlinear Klein-Gordon Equation
}

\author{
Maryam Sarboland ${ }^{1}$, Azim Aminataei ${ }^{2}$ \\ ${ }^{1}$ Department of Mathematics, Saveh Branch, Islamic Azad University, P.O. Box: 39187-366, Saveh, Iran, \\ m.sarboland@gmail.com \\ ${ }^{2}$ Faculty of Mathematics, Department of Applied Mathematics, K. N. Toosi University of Technology, \\ P.O. Box: 15418-49611, Tehran, Iran. \\ ataei@kntu.ac.ir
}

Article history:

Received November 26, 2014

Accepted January 4, 2015

Available online January 6, 2015

\begin{abstract}
A collocation scheme based on the use of the multiquadric quasi-interpolation operator $L_{W_{2}}$, integrated radial basis function networks (IRBFNs) method and three order finite difference method is applied to the nonlinear Klein-Gordon equation. In the present scheme, the three order finite difference method is used to discretize the temporal derivative and the integrated form of the multiquadric quasi-interpolation scheme is used to approximate the unknown function and its spatial derivatives. Several numerical experiments are provided to show the efficiency and the accuracy of the given method.
\end{abstract}

Keywords: Nonlinear Klein-Gordon equation; multiquadric quasi-interpolation scheme; collocation scheme; integrated radial basis function networks.

\section{Introduction}

The Klein-Gordon equation is a relativistic version of the Schrodinger equation, which describes scalar spineless particles. It appears in various physical applications such as the propagation of fluxions in the Josephson junctions, the motion of rigid pendula attached a stretched wire, and dislocations in crystals [21].

In this paper, we consider the one-dimensional nonlinear Klein-Gordon equation of the form: 
$u_{t t}+\mu u_{x x}+F(u)=f(x, t), \quad x \in \Omega=[a, b] \subset \mathbb{R}, 0<t \leqslant T$,

with the initial conditions

$u(x, 0)=g_{1}(x), \quad x \in \Omega$,
$u_{1}(x, 0)=g_{2}(x), \quad x \in \Omega$,

and the boundary condition

$u(x, t)=g(x, t), \quad x \in \partial \Omega$,

where $u=u(x, t)$ represents the wave displacement at position $x$ and time $t, \mu$ is a known constant, $F(u)$ is the nonlinear force such that $\frac{\partial F}{\partial u} \geqslant 0$ and $g_{1}(x), g_{2}(x)$ and $g(x, t)$ are known functions.

The general form of (1) covers many different generalized Klein-Gordon equations arising in various physical applications. For example, when $F(u)=\sin (u), \quad F(u)=\sin (u)+\sin (2 u)$ and $F(u)=\sinh (u)+\sinh (2 u)$, (1) becomes the well-known sine-Gordon equation, the double sine-Gordon equation and the double sinh-Gordon equation, respectively. The above nonlinear Klein-Gordon equations are Hamiltonian partial differential equations (PDEs), and for a wide class of force $F(u)$, it has the conserved Hamiltonian quantity (or energy) [18]

$H=\int\left(\frac{1}{2} u_{t}^{2}+\frac{1}{2} u_{x}^{2}+G(u)\right) d x$,

where $\mathrm{G}^{\prime}(\mathrm{u})=\mathrm{F}(\mathrm{u})$.

Up to now, many authors have studied the numerical and approximation solutions of the nonlinear Klein-Gordon equation by using various techniques that some of them are the finite difference method, the spectral method, the adomian decomposition method, the inverse scattering method, Bäcklund transformation, the auxiliary equation method, the Wadatitrace method, Hirota bilinear forms, pseudospectral method, the tanh-sech method, the sine-cosine method, Jacobi elliptic functions and the Riccati equation expansion method and so on (see $[10,15,25,26,30,31]$ and references therein).

Finite difference methods are known as the first technique for solving PDEs. Even though these methods have been widely used but they require the construction and update of a mesh and hence bring inconvenience during computation.

In 1990, Kansa [13, 14] modified Hardy's multiquadric (MQ) RBF method to solve PDEs. Since then, using of the RBFs collocation method for solving of PDEs have attracted the attention of researchers because no tedious mesh generation is required. Recently, the Kansa's method has been developed to simulate nonlinear PDEs [2] such as Burger's equation [11], sine-Gordon equation [6], Klein-Gordon equation [7], and Korteweg-de Vries (KdV) equation [5] etc. In all these works, the solution function is decomposed into RBFs and its derivatives are then obtained through differentiation that caused the reduction in convergence rate. In order to avoid from this problem, Mai-Duy and Tran-Cong introduced an integrated MQ-RBFNs scheme for the approximation of function and its derivatives [20]. Numerical 
experiments and theoretical analysis indicate that for solving PDEs integrated RBF (IRBF) procedure is more accurate in comparison with direct RBF (DRBF) procedure. Also, IRBF scheme is more stable than DRBF for a range of PDEs [20, 27].

In both DRBF and IRBF schemes, one must resolve a linear system of equations at each time step. Hon and $\mathrm{Wu}[12], \mathrm{Wu}[8,9]$ and others have provided some successful examples using MQ quasiinterpolation scheme for solving differential equations. Beatson and Powell [1] and Wu and Schaback [29] proposed other univariate MQ quasi-interpolations. In [3, 4], Chen and Wu used MQ quasiinterpolation to solve Burgers' equation and hyperbolic conservation laws. Also, Xiao et al. [25] presented the numerical method based on Chen and Wu's method for solving the third-order KdV equation. Recently, Jiang et al. [16] have introduced a new multi-level univariate MQ quasi-interpolation approach with high approximation order compared with initial MQ quasi-interpolation scheme. This approach is based on inverse multiquadric (IMQ) RBF interpolation, and Wu and Schaback's MQ quasi-interpolation operator $L_{D}$ that have the advantages of high approximation order.

The Jiang et al. MQ quasi-interpolation one-dimensional operator $L_{W_{2}}$ is summation of two series that the second series coefficients are combined of first series coefficients. By giving relation between two series coefficients based on function values, we can convert it to a compact form that is based on one series and can use it in integrated form for the numerical solution of PDEs.

This paper presents a novel numerical scheme to solve the nonlinear Klein-Gordon equation that is based on integrated MQ quasi-interpolation scheme. The rest of present paper is arranged as follows. Brief information of the MQ quasi-interpolation scheme is given in Section 2. Section 3 describes our method on the nonlinear Klein-Gordon equation. Several numerical experiments are presented in Section 4, followed by a conclusion summary in Section 5.

\section{The MQ quasi-interpolation scheme}

In this section, some elementary knowledge about of three univariate MQ quasi-interpolation schemes, namely, $L_{D}, L_{W}$ and $L_{W_{2}}$ is presented. For more information about MQ quasi-interpolation operators see $[1,3,4,29]$.

For a given interval $\Omega=[a, b]$ and a finite set of distinct points

$a=x_{0}<x_{1}<\ldots<x_{N}=b, \quad h=\max _{1 \leqslant i \leqslant N}\left(x_{i}-x_{i-1}\right)$,

quasi-interpolation of a univariate function $f:[a, b] \rightarrow \mathbb{R}$ is given by

$L(f)=\sum_{i=0}^{N} f\left(x_{i}\right) \phi_{i}(x)$

where function $\phi_{i}(x)$ is a linear combination of the MQs

$\psi_{i}(x)=\sqrt{c^{2}+\left(x-x_{i}\right)^{2}}$ 
and $c \in \mathbb{R}^{+}$is a shape parameter. In [29], Wu and Scheback presented the univariate MQ quasiinterpolation operator $\mathrm{L}_{\mathbf{D}}$ that is defined as

$L_{D} f(x)=\sum_{i=0}^{N} f\left(x_{i}\right) \psi_{i}(x)$

where

$\psi_{0}(x)=\frac{1}{2}+\frac{\psi_{1}(x)-\left(x-x_{0}\right)}{2\left(x_{1}-x_{0}\right)}$

$\psi_{1}(x)=\frac{\psi_{2}(x)-\psi_{1}(x)}{2\left(x_{2}-x_{1}\right)}-\frac{\psi_{1}(x)-\left(x-x_{0}\right)}{2\left(x_{1}-x_{0}\right)}$,

$\psi_{i}(x)=\frac{\psi_{i+1}(x)-\psi_{i}(x)}{2\left(x_{i+1}-x_{i}\right)}-\frac{\psi_{i}(x)-\psi_{i-1}(x)}{2\left(x_{i}-x_{i-1}\right)}, \quad 2 \leqslant i \leqslant N-2$,

$\psi_{N-1}(x)=\frac{\left(x_{N}-x\right)-\psi_{N-1}(x)}{2\left(x_{N}-x_{N-1}\right)}-\frac{\psi_{N-1}(x)-\psi_{N-2}(x)}{2\left(x_{N-1}-x_{N-2}\right)}$,

and

$\psi_{N}(x)=\frac{1}{2}+\frac{\psi_{N-1}(x)-\left(x_{N}-x\right)}{2\left(x_{N}-x_{N-1}\right)}$.

In RBFs interpolation, high approximation order can be gotten by increasing the number of interpolation centers but we have to solve unstable linear system of equations. By using MQ quasiinterpolation scheme, we can avoid this problem, whereas the approximation order is not good. Therefore, Jiang et al. [16] defined two MQ quasi-interpolation operators denoted as $L_{W}$ and $L_{W_{2}}$ which pose the advantages of RBFs interpolation and MQ quasi-interpolation scheme. The process of MQ quasiinterpolation of $L_{W}$ and $L_{W_{2}}$ are as follows that is described in [16].

Suppose that $\left\{x_{k_{j}}\right\}_{j=1}^{\bar{N}}$ is a smaller set from the given points $\left\{x_{i}\right\}_{i=0}^{N}$ where $\bar{N}$ is a positive integer satisfying $\bar{N}<N$ and $0=k_{0}<k_{1}<\ldots<k_{\bar{N}+1}=N$. Using the IMQ-RBF, the second derivative of $f(x)$ can be approximated by RBF interpolant $S_{f^{\prime \prime}}$ as

$S_{f^{\prime \prime}}(x)=\sum_{j=1}^{\bar{N}} \alpha_{j} \bar{\varphi}_{j}(x)$

where 
$\bar{\varphi}_{j}(x)=\frac{s^{2}}{\left(s^{2}+\left(x-x_{k_{j}}\right)^{2}\right)^{3 / 2}}$

and $s \in \mathbb{R}^{+}$is a shape parameter. The coefficients $\left\{\alpha_{j}\right\}_{j=1}^{\bar{N}}$ are uniquely determined by the interpolation condition

$S_{f^{\prime \prime}}\left(x_{k_{i}}\right)=\sum_{j=1}^{\bar{N}} \alpha_{j} \bar{\varphi}_{j}\left(x_{k_{i}}\right)=f^{\prime \prime}\left(x_{k_{i}}\right), \quad 1 \leqslant i \leqslant \bar{N}$

Since, the Eq. (7) is solvable [19], so

$\alpha=A_{X}^{-1} f_{X}^{\prime \prime}$,

where

$$
X=\left\{x_{k_{1}}, \ldots, x_{k_{\bar{N}}}\right\}, \quad \alpha=\left[\alpha_{1}, \ldots, \alpha_{\bar{N}}\right]^{T}, \quad A_{X}=\left[\bar{\varphi}_{j}\left(x_{k_{i}}\right)\right], \quad f_{X}^{\prime \prime}=\left[f^{\prime \prime}\left(x_{k_{1}}\right), \ldots, f^{\prime \prime}\left(x_{k_{\bar{N}}}\right)\right]^{T}
$$

By using $f$ and the coefficient $\alpha$ defined in Eq. (8), a function $e(x)$ is constructed in the form

$$
e(x)=f(x)-\sum_{i=1}^{\bar{N}} \alpha_{i} \sqrt{s^{2}+\left(x-x_{k_{i}}\right)^{2}} .
$$

Then the MQ quasi-interpolation operator $L_{W}$ by using $L_{D}$ defined by Eqs. (4) and (5) on the data $\left(x_{i}, e\left(x_{i}\right)\right)_{0 \leqslant i \leqslant N}$ with the shape parameter $c$ is defined as follows:

$L_{W} f(x)=\sum_{i=1}^{\bar{N}} \alpha_{i} \sqrt{s^{2}+\left(x-x_{k_{i}}\right)^{2}}+L_{D} e(x)$

The shape parameters $c$ and $s$ should not be the same constants in the Eq. (10).

In Eq. (7), $f_{x_{k_{j}}}^{\prime \prime}$ can be replaced by

$f_{x_{k_{j}}}^{\prime \prime}=\frac{2\left[\left(x_{k_{j}}-x_{k_{j-1}}\right) f\left(x_{k_{j+1}}\right)-\left(x_{k_{j+1}}-x_{k_{j-1}}\right) f\left(x_{k_{j}}\right)+\left(x_{k_{j+1}}-x_{k_{j}}\right) f\left(x_{k_{j-1}}\right)\right]}{\left(x_{k_{j}}-x_{k_{j-1}}\right)\left(x_{k_{j+1}}-x_{k_{j}}\right)\left(x_{k_{j+1}}-x_{k_{j-1}}\right)}$,

when the data $\left(x_{k_{i}}, f\left(x_{k_{i}}\right)\right)_{0 \leqslant i \leqslant \bar{N}}$ are given. So, if $f_{X}^{\prime \prime}$ in Eq. (8) replace by

$F_{X}^{\prime \prime}=\left(f_{x_{k_{1}}}^{\prime \prime}, \ldots, f_{x_{k_{\bar{N}}}^{\prime \prime}}^{\prime \prime}\right)^{T}$

the quasi-interpolation operator defined by Eqs. (9) and (10) is denoted by $L_{W_{2}}$. For more details about 
the properties and accuracy of $L_{W}$ and $L_{W_{2}}$, one can see [26]. In this paper, we use the MQ quasiinterpolation operator $L_{W_{2}}$ with equally spaced points and $N=2 \bar{N}$.

The operator $L_{W_{2}}$ can be written in the compact form

$L_{W_{2}} f(x)=\sum_{i=0}^{N} f\left(x_{i}\right) \psi_{i}(x)$

where the basis functions $\psi_{i}(x)$ are obtained by substituting Equations (8), (9) and (11) into (10), see [23].

By writing operator $L_{W_{2}}$ in the compact form (12), we can use it in the indirect form for the numerical solution of PDEs. Also, by converting operator $L_{W_{2}}$ to form (12), we do not require to solve a linear system of equations for getting of the coefficients $\alpha_{i}$ at each time step, see [17].

\section{The numerical method}

In this section, the numerical scheme is presented for solving the Klein-Gordon equation (1) by using the MQ quasi-interpolation $L_{W_{2}}$. In our approach, the fourth order finite difference approximation is first employed for discretizing of the temporal derivative similar to work that Rashidinia did in [22]. Then, the highest order derivatives (second order in this paper) of the solution function are approximated by Eq. (12), and their lower order derivatives and the solution function are then obtained by symbolic integration. At the end, the collocation scheme is applied.

\subsection{The discretization of time}

According of the fourth order finite difference, the term $u_{t t}^{n}=u_{t t}\left(x, t_{n}\right), t_{n}=n \Delta t$ can be arranged as

$u_{t t}^{n} \cong \frac{\delta_{t}^{2}}{(\Delta t)^{2}\left(1+\delta_{t}^{2}\right)} u^{n}+O\left((\Delta t)^{4}\right)$,

where $\delta_{t}^{2}=u^{n+1}-2 u^{n}+u^{n-1}$.

Substituting Eq. (13) into Eq. (1) yields the following time discretized form of Klein-Gordon equation:

$\delta_{t}^{2} u^{n}+\mu(\Delta t)^{2}\left(1+\gamma \delta_{t}^{2}\right) u_{x x}^{n}+(\Delta t)^{2}\left(1+\gamma \delta_{t}^{2}\right) F^{n}=(\Delta t)^{2}\left(1+\gamma \delta_{t}^{2}\right) f^{n}$,

where $f^{n}=f\left(x, t_{n}\right)$ and $F^{n}=F\left(u^{n}\right)$.

In this paper, we consider the nonlinear force $F(u)=\alpha u+\beta u^{k}$ wherein $k=2$ or $k=3$ and $\alpha$ and $\beta$ are known constants. After some arrangements, Eq. (14) can be written in the following form: 


$$
\left(\frac{1}{\gamma(\Delta t)^{2}}+\alpha\right) u^{n+1}+\mu u_{x x}^{n+1}+\beta\left(u^{n+1}\right)^{k}=\chi(x)
$$

where

$\chi(x)=\left(\frac{2}{\gamma(\Delta t)^{2}}-\alpha \kappa\right) u^{n}-\left(\frac{1}{\gamma(\Delta t)^{2}}+\alpha\right) u^{n-1}-\mu\left[u_{x x}^{n}+u_{x x}^{n-1}\right]-\beta\left[\kappa\left(u^{n}\right)^{k}+\left(u^{n-1}\right)^{k}\right]$

and $\kappa=\frac{1-2 \gamma}{\gamma}$.

\subsection{The indirect MQ quasi-interpolation scheme}

In this scheme, the highest order derivatives (second order in this paper) of the solution function, $u_{x x}^{n}$, is approximated by MQ quasi-interpolation $L_{W_{2}}$ on data $\left\{x_{j}\right\}_{j=1}^{N-1}$ as follows:

$u_{x x}^{n}(x)=\sum_{j=1}^{N-1} u_{x x}^{n}\left(x_{j}\right) \hat{\psi}_{j}(x)$

Now, symbolic integrating of Eq. (16) yields

$u_{x}^{n}(x)=\sum_{j=1}^{N-1} u_{x x}^{n}\left(x_{j}\right) \int \hat{\psi}_{j}(x) d x+C_{1}$,

$u^{n}(x)=\sum_{j=1}^{N-1} u_{x x}^{n}\left(x_{j}\right) \iint \hat{\psi}_{j}(x) d x d x+C_{1} x+C_{2}$

The Eqs. (16)- (18) can be rewritten in the compact form as follows:

$u^{n}(x)=\sum_{j=0}^{N} w_{j}^{n} \varphi_{j}(x), \quad u_{x}^{n}(x)=\sum_{j=0}^{N} w_{j}^{n} \dot{\varphi}_{j}(x), \quad u_{x x}^{n}(x)=\sum_{j=0}^{N} w_{j}^{n} \ddot{\varphi}_{j}(x)$,

where

$\varphi_{j}(x)=\iint \hat{\psi}_{j}(x) d x d x, \quad 1 \leqslant j \leqslant N-1, \quad \varphi_{0}(x)=x, \quad \varphi_{N}(x)=1$,

$\dot{\varphi}_{j}(x)=\int \hat{\psi}_{j}(x) d x, \quad 1 \leqslant j \leqslant N-1, \quad \dot{\varphi}_{0}(x)=1, \quad \dot{\varphi}_{N}(x)=0$,

$\ddot{\varphi}_{j}(x)=\hat{\psi}_{j}(x), \quad 1 \leqslant j \leqslant N-1, \quad \ddot{\varphi}_{0}(x)=0, \quad \ddot{\varphi}_{N}(x)=0$,

and 
$w_{j}^{n}=u_{x x}^{n}\left(x_{j}\right), \quad 1 \leqslant j \leqslant N-1, w_{0}^{n}=C_{1}, w_{N}^{n}=C_{2}$.

Now, substituting Eq. (19) into Eqs. (15) and (3) and applying collocation method yield the following equations:

$$
\left(\frac{1}{\gamma(\Delta t)^{2}}+\alpha\right) \sum_{j=0}^{N} w_{j}^{n+1} \varphi_{i j}+\mu \sum_{j=0}^{N} w_{j}^{n+1} \ddot{\varphi}_{i j}+\beta\left(\sum_{j=0}^{N} \tilde{w}_{j}^{n+1} \varphi_{i j}\right)^{k}=\chi\left(x_{i}\right), \quad i=1, \ldots, N-1,
$$

and

$$
\sum_{j=0}^{N} w_{j}^{n+1} \varphi_{i j}=g^{n+1}\left(x_{i}\right), \quad i=0, N,
$$

where $\varphi_{i j}=\varphi_{j}\left(x_{i}\right), \ddot{\varphi}_{i j}=\ddot{\varphi}_{j}\left(x_{i}\right), g^{n}\left(x_{i}\right)=g\left(x_{i}, t_{n}\right)$ and

$\chi\left(x_{i}\right)=\left(\frac{2}{\gamma(\Delta t)^{2}}-\alpha \kappa\right) u^{n}\left(x_{i}\right)-\left(\frac{1}{\gamma(\Delta t)^{2}}+\alpha\right) u^{n-1}\left(x_{i}\right)-\mu\left[u_{x x}^{n}\left(x_{i}\right)+u_{x x}^{n-1}\left(x_{i}\right)\right]-\beta\left[\kappa\left(u^{n}\left(x_{i}\right)\right)^{k}+\left(u^{n-1}\left(x_{i}\right)\right)^{k}\right]$.

At $n=1$, according to the initial conditions that was introduced in (2) and approach that Rashidinia did in [30] on based Taylor series, we apply the following assumptions

$u^{0}(x)=u(x, 0)=g_{1}(x)$,

and

$u^{1}(x)=g_{1}(x)+\Delta g_{2}(x)-\frac{(\Delta t)^{2}}{2 !}\left[\mu u_{x x}+F-f\right]^{0}-\frac{(\Delta t)^{3}}{3 !}\left[\mu g_{2}^{\prime \prime}(x)+F_{t}-f_{t}+F_{u} u_{t}\right]^{0}+\mu \frac{(\Delta t)^{4}}{4 !}\left[\mu u_{x x x x}\right.$

$\left.+F_{x} u_{x}+F_{x x}-f_{x x}+F_{x u} u_{x}+u_{x}\left(F_{x u}+F_{u u} u_{x}\right)\right]^{0}+O\left((\Delta t)^{5}\right)$,

In each time step (for example time step $n+1$ ), at first we set $\tilde{w}_{j}^{n+1}=w_{j}^{n}$. Having this, Eqs. (20) and (21) are solved as a system of linear algebraic equations for unknowns $w_{j}^{n+1} ; j=0,1, \ldots, N$. Then, we recomputed $\tilde{w}_{j}^{n+1}=w_{j}^{n+1}$ where $w_{j}^{n+1}$ as we illustrate, can be obtained by solving Eqs. (20) and (21). Now, at each time level, we iterate calculating $\tilde{w}_{j}^{n+1}$ and solving the approximation values of the unknown, until the tolerance of any two latest iterations is not bigger than $10^{-8}$, i.e. a predictor-corrector scheme is adopted in each time level, then we can move on to the next time level. 


\section{The numerical experiments}

Four experiments are studied to investigate the robustness and the accuracy of the proposed method. The numerical results of the Klein-Gordon equation by using this scheme is compared with the analytical solutions and solutions in [7, 22]. These methods include Thin Plate Splines (TPS) RBF collocation method [7] and cubic B-spline collocation method (CBS) [22]. Our scheme is denoted by IMQQI. The $\mathrm{L}_{2}, \mathrm{~L}_{\infty}$ and RMS error norms which are defined by

$$
\begin{aligned}
& \mathrm{L}_{2} \# \mathrm{u}^{* n}-u^{n} \|_{2}=\sqrt{h \sum_{j=0}^{N}\left(u^{* n}\left(x_{j}\right)-u^{n}\left(x_{j}\right)\right)^{2}}, \\
& \mathrm{~L}_{\infty} \# \mathrm{u}^{* n}-u^{n} \|_{\infty}=\max _{0 \leqslant j \leqslant N}\left|u^{* n}\left(x_{j}\right)-u^{n}\left(x_{j}\right)\right| \\
& \mathrm{RMS}=\sqrt{\left(\sum_{j=0}^{N}\left(u^{* n}\left(x_{j}\right)-u^{n}\left(x_{j}\right)\right)^{2}\right) /(N+1)},
\end{aligned}
$$

are used to measure the accuracy of our scheme where $u^{*}$ is the approximation solution. In all experiments, the shape parameter $s$ is considered twice of the shape parameter $c$.

The computations associated with our experiments are performed in Maple 14 on a PC with a CPU of 2.4 GHZ.

Experiment 1. In this experiment, the Klein-Gordon equation (1) is considered with $\mu=1$, $f(x, t)=-x \cos (t)+x^{2} \cos (t)$ in interval $-1 \leqslant x \leqslant 1$ and the nonlinear force $F(u)=u^{2}$ so the values of constants in (15) are $\alpha=0, \beta=1$ and $k=2$. The initial conditions are given by

$u(x, 0)=x, \quad-1 \leqslant x \leqslant 1$,

$u_{t}(x, 0)=0, \quad-1 \leqslant x \leqslant 1$.

The exact solution is given in [28] as

$u(x, t)=x \cos (t)$.

The boundary function $g(x, t)$ can be extracted from the exact solution. The $\mathrm{L}_{2}, \mathrm{~L}_{\infty}$ and RMS errors in the solutions with $\Delta t=0.0001, N=10, c=1.63 \times 10^{-2}$ and $\gamma=\frac{1}{12}$ that calculated in 100 points are listed in Table 1 and compared with the results in [7,22].

The space-time graphs of the estimated solution are drawn in Fig. 1. Table 1 indicates that the proposed method requires more less nodes to attain the accuracy of the CBSM [22] and TPSM [7]. Also, it shows that this scheme performs better than TPSM. 


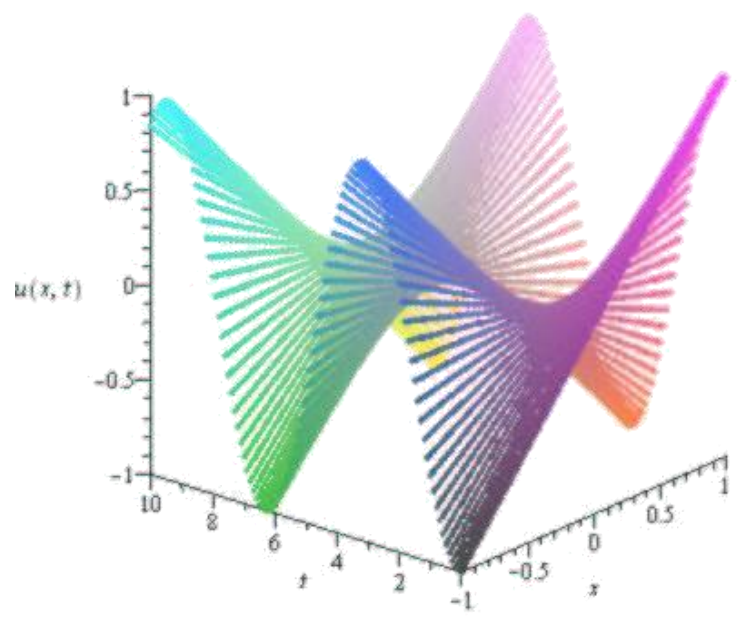

Figure 1: The graph of the estimated solution up to $t=10$ with $\Delta t=0.0001$ and $N=10$ of experiment 1.

Table 1: The comparison of the $L_{1} ; L_{2}$ and RMS errors of our method with the results of $[7,22]$ at different times of experiment 1.

\begin{tabular}{|c|c|c|c|c|c|}
\hline Time & 1 & 3 & 5 & 7 & 10 \\
\hline \multicolumn{6}{|c|}{ IMQQI; N = 10} \\
\hline $\mathrm{L}_{1}$ & $9.6719 \mathrm{E}-11$ & $1.4351 \mathrm{E}-10$ & $1.7096 \mathrm{E}-10$ & $1.4199 \mathrm{E}-10$ & $1.0761 \mathrm{E}-10$ \\
\hline $\mathrm{L}_{2}$ & $6.6578 \mathrm{E}-11$ & $1.1603 \mathrm{E}-10$ & $1.5434 \mathrm{E}-10$ & $1.4325 \mathrm{E}-10$ & $7.6498 \mathrm{E}-11$ \\
\hline RMS & $4.6771 \mathrm{E}-11$ & $8.1926 \mathrm{E}-11$ & $1.0882 \mathrm{E}-10$ & $1.0164 \mathrm{E}-10$ & $5.3802 \mathrm{E}-11$ \\
\hline Time & 1 & 3 & 5 & 10 & 20 \\
\hline \multicolumn{6}{|c|}{ CBSM [22]; $\mathrm{N}=100$} \\
\hline $\mathrm{L}_{1}$ & $4.7698 \mathrm{E}-13$ & $2.8899 \mathrm{E}-13$ & $4.3667 \mathrm{E}-13$ & $8.4593 \mathrm{E}-13$ & $3.1344 \mathrm{E}-12$ \\
\hline $\mathrm{L}_{2}$ & $1.8986 \mathrm{E}-12$ & $1.0680 \mathrm{E}-12$ & $1.5686 \mathrm{E}-12$ & $5.3244 \mathrm{E}-12$ & 4.4324E-11 \\
\hline RMS & $2.6850 \mathrm{E}-14$ & $1.5105 \mathrm{E}-13$ & $2.2184 \mathrm{E}-12$ & $5.9738 \mathrm{E}-12$ & $2.2332 \mathrm{E}-12$ \\
\hline Time & 1 & 3 & 5 & 7 & 10 \\
\hline \multicolumn{6}{|c|}{ TPSM [7]; $N=100$} \\
\hline L1 & $1.2540 \mathrm{E}-05$ & $1.5554 \mathrm{E}-05$ & $3.3792 \mathrm{E}-05$ & $3.7753 \mathrm{E}-05$ & $1.3086 \mathrm{E}-05$ \\
\hline $\mathrm{L}_{2}$ & $6.5422 \mathrm{E}-05$ & 1.1717E-04 & $2.2011 \mathrm{E}-04$ & $2.5892 \mathrm{E}-04$ & 7.9854E-05 \\
\hline RMS & $6.5097 \mathrm{E}-06$ & $1.1659 \mathrm{E}-05$ & $2.1902 \mathrm{E}-05$ & $2.5763 \mathrm{E}-05$ & $7.9458 \mathrm{E}-06$ \\
\hline
\end{tabular}

Experiment 2. Consider the Klein-Gordon equation (1) with $\mu=1, f(x, t)=6 x t\left(x^{2}-t^{2}\right)+x^{6} t^{6}$ in interval $0 \leqslant x \leqslant 1$ and the nonlinear force $F(u)=u^{2}$ wherein $\alpha, \beta$ and $k$ are considered 0,1 and 2, respectively. The initial conditions are given by

$$
\begin{aligned}
& u(x, 0)=0, \quad 0 \leqslant x \leqslant 1, \\
& u_{t}(x, 0)=0, \quad 0 \leqslant x \leqslant 1 .
\end{aligned}
$$


The exact solution is given in [28] as

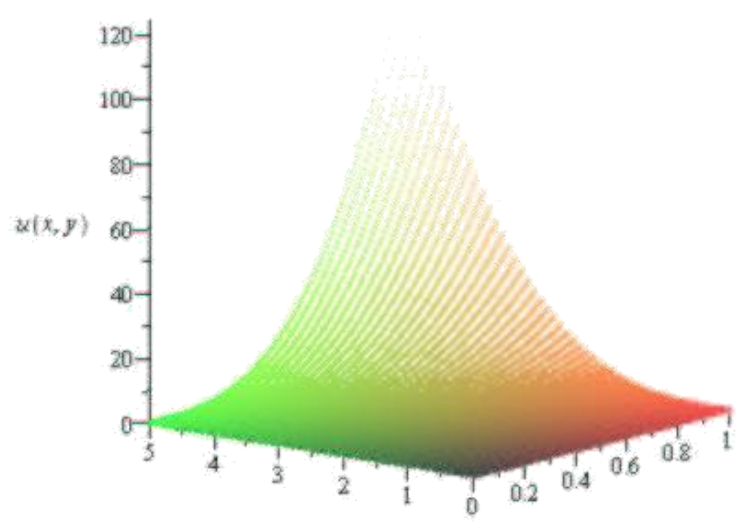

Figure 2: The graph of the estimated solution up to $t=5$ with $\Delta t=0.001$ and $N=50$ of experiment 2 .

Table 2: The comparison of the $L_{1} ; L_{2}$ and RMS errors of our method with the results of $[7,22]$ at different times of experiment 2.

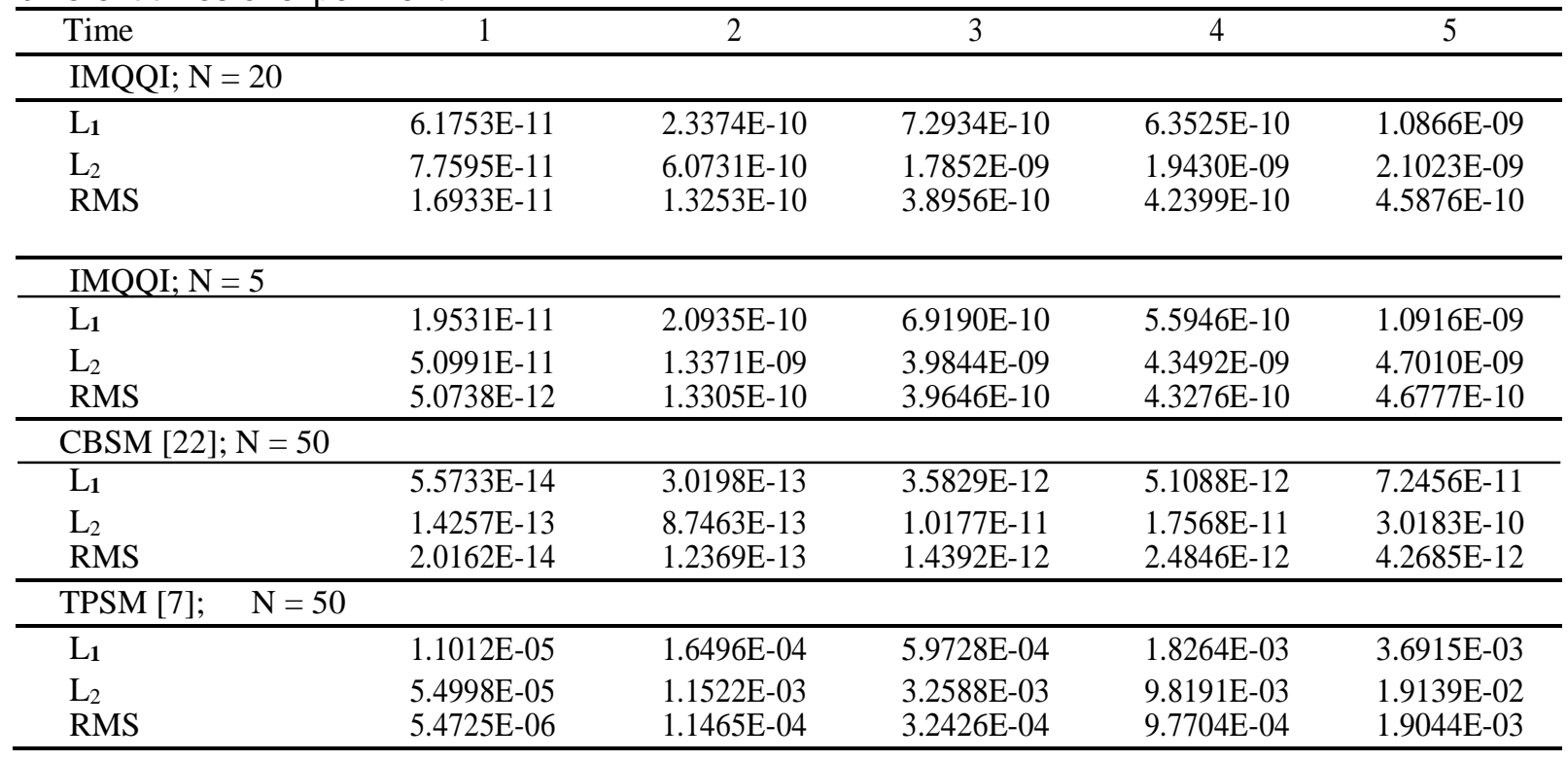

$u(x, t)=x^{3} t^{3}$.

The boundary function $g(x, t)$ can be extracted from the exact solution. Table 2 shows the $\mathrm{L}_{2}, \mathrm{~L}_{\infty}$ and RMS errors in the solutions with $\Delta t=0.001, N=50, c=1.63 \times 10^{-2}$ and $\gamma=\frac{1}{12}$. Our numerical results are compared with the results in $[7,22]$. Moreover, the space-time graph of the estimated solution is drawn in Fig. 2. 
Table 2 indicates that the proposed method requires more less nodes to attain the accuracy of the TPSM [7] and has better accuracy than TPSM [7].
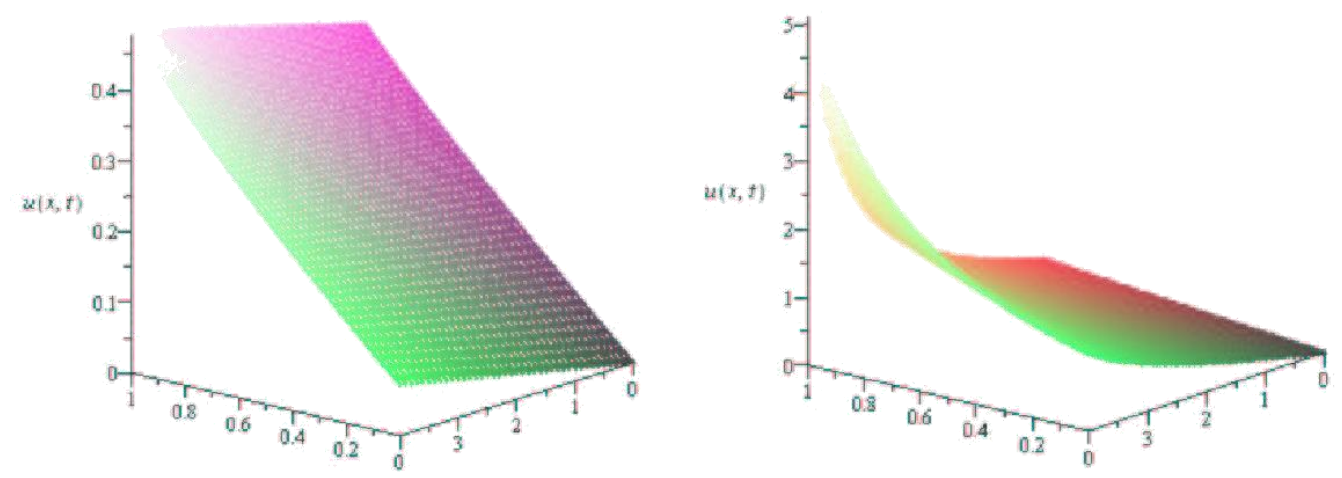

Figure 3: The graph of the estimated solution up to $t=4$ with $\Delta t=0.001$ and $N=30$ of experiment 3, (Left) $C=0.05$, (Right) $C=0.5$.

Table 3: The comparison of the $\mathrm{L}_{\infty}, \mathrm{L}_{2}$ and RMS errors of our method with the results of $[7,22]$ with $C=0.05$ at different times of experiment 3 .

\begin{tabular}{lcccc}
\hline Time & 1 & 2 & 3 & 4 \\
\hline IMQQI; N = 50 & & & & \\
\hline L1 & $3.2112 \mathrm{E}-09$ & $3.5349 \mathrm{E}-09$ & $4.0395 \mathrm{E}-09$ & $4.7216 \mathrm{E}-09$ \\
$\mathrm{~L}_{2}$ & $1.1963 \mathrm{E}-08$ & $1.6133 \mathrm{E}-08$ & $1.8570 \mathrm{E}-08$ & $1.7156 \mathrm{E}-08$ \\
RMS & $1.6752 \mathrm{E}-09$ & $2.2591 \mathrm{E}-09$ & $2.6003 \mathrm{E}-09$ & $2.4023 \mathrm{E}-09$ \\
\hline IMQQI; N = 100 & & & & \\
\hline L1 & $1.9972 \mathrm{E}-10$ & $2.2253 \mathrm{E}-10$ & $2.4427 \mathrm{E}-10$ & $2.8106 \mathrm{E}-10$ \\
$\mathrm{~L}_{2}$ & $8.6479 \mathrm{E}-10$ & $8.2885 \mathrm{E}-10$ & $9.3908 \mathrm{E}-10$ & $1.1417 \mathrm{E}-09$ \\
RMS & $8.6050 \mathrm{E}-11$ & $8.2474 \mathrm{E}-11$ & $9.3442 \mathrm{E}-11$ & $1.1360 \mathrm{E}-10$ \\
\hline CBSM [22]; N =100 & & & \\
\hline L1 & $1.1986 \mathrm{E}-08$ & $2.4733 \mathrm{E}-08$ & $2.8958 \mathrm{E}-08$ & $1.9916 \mathrm{E}-08$ \\
L 2 & $7.5619 \mathrm{E}-08$ & $1.7997 \mathrm{E}-07$ & $2.0797 \mathrm{E}-07$ & $1.4058 \mathrm{E}-07$ \\
RMS & $7.5619 \mathrm{E}-08$ & $1.7997 \mathrm{E}-08$ & $2.0797 \mathrm{E}-08$ & $1.4058 \mathrm{E}-08$ \\
\hline TPSM [7]; N = 100 & & & & \\
\hline L1 & $3.6497 \mathrm{E}-07$ & $3.8952 \mathrm{E}-07$ & $4.2123 \mathrm{E}-07$ & $4.5928 \mathrm{E}-07$ \\
$\mathrm{~L}_{2}$ & $1.7861 \mathrm{E}-06$ & $1.5383 \mathrm{E}-06$ & $1.7275 \mathrm{E}-06$ & $2.0097 \mathrm{E}-06$ \\
RMS & $1.7772 \mathrm{E}-07$ & $1.5306 \mathrm{E}-07$ & $1.7190 \mathrm{E}-07$ & $1.9997 \mathrm{E}-07$ \\
\hline
\end{tabular}

Experiment 3. In this experiment, the Klein-Gordon equation (1) is considered with $\mu=2.5, f(x, t)=0$ in interval $0 \leqslant x \leqslant 1$ and the nonlinear force $F(u)=u+1.5 u^{3}$ wherein $\alpha, \beta$ and $k$ are $1,1.5$ and 3 , respectively. The initial conditions are given by

$u(x, 0)=B \tan (K x), \quad 0 \leqslant x \leqslant 1$,

$u_{t}(x, 0)=B C K \sec ^{2}(K x), \quad 0 \leqslant x \leqslant 1$. 
The exact solution is given in [32] as

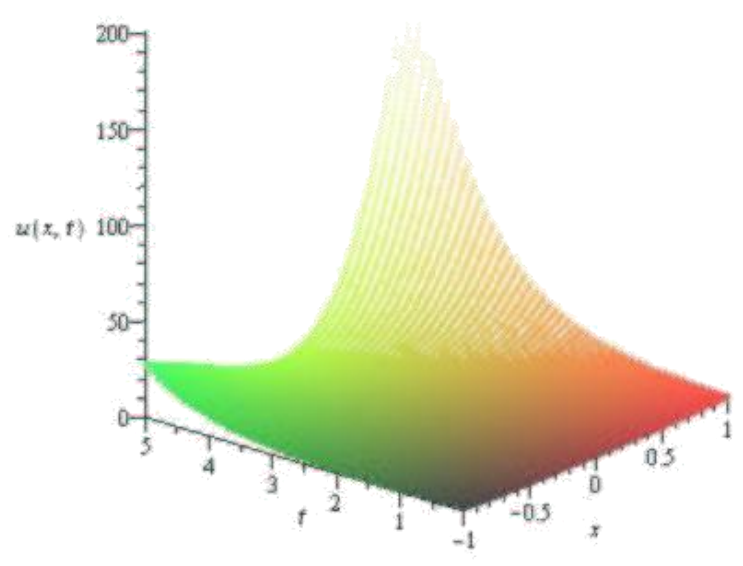

Figure 4: The graph of the estimated solution up to $t=5$ with $\Delta t=0.001$ and $N=50$ of experiment 4 .

Table 4: The comparison of the $\mathrm{L}_{\infty}, \mathrm{L}_{2}$ and RMS errors of our method with the results of $[7,22]$ with $C=0.5$ at different times of experiment 3 .

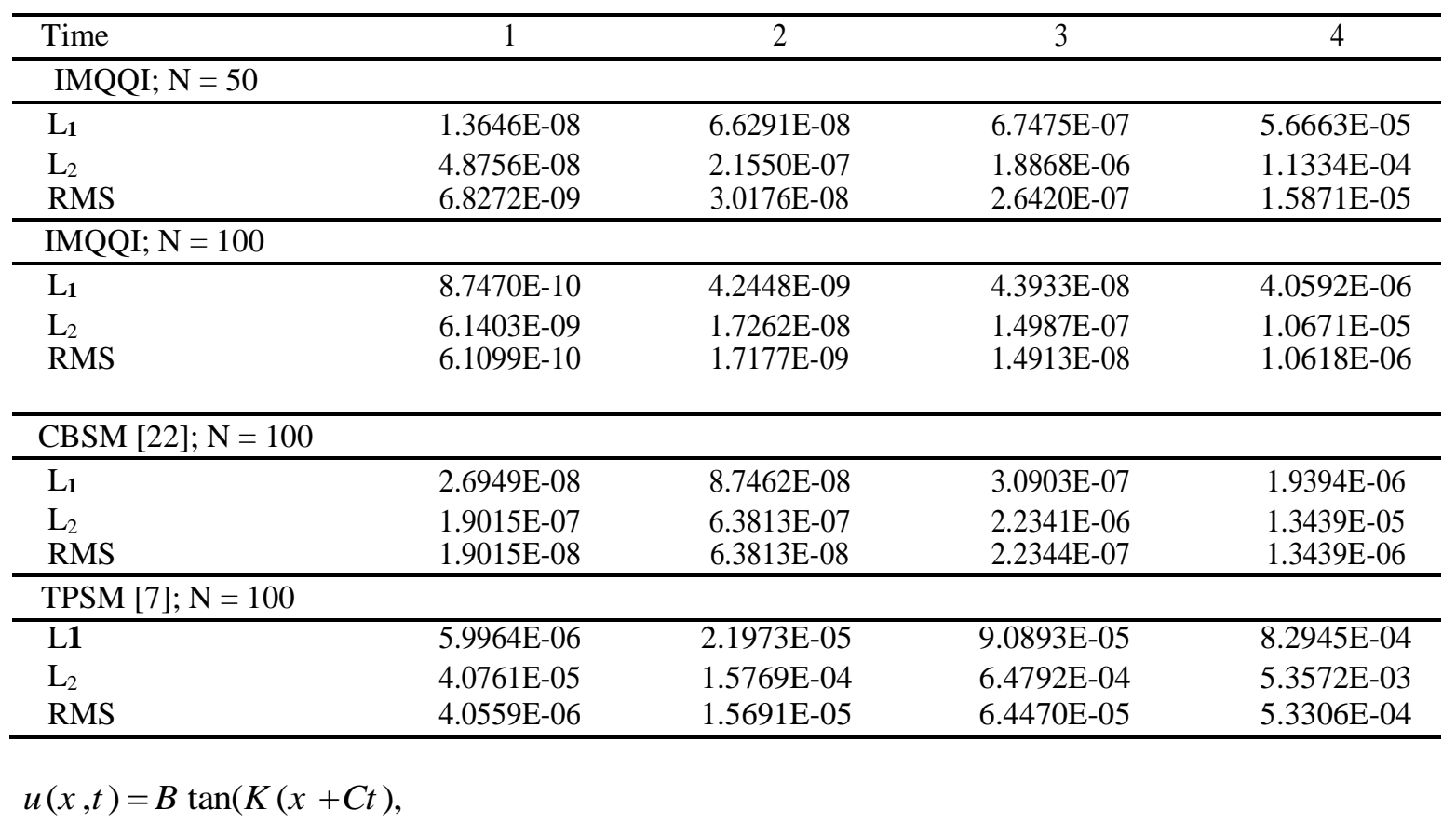


where $B=\sqrt{\frac{\alpha}{\beta}}$ and $K=\sqrt{\frac{\alpha}{-2 \mu+C^{2}}}$. The boundary function $g(x, t)$ can be extracted from the exact solution. In Tables 3 and 4 , the $\mathrm{L}_{2}, \mathrm{~L}_{\infty}$ and RMS errors in the solutions are listed with $\Delta t=0.0001$, $N=100, N=50, \gamma=\frac{1}{12}$ and $C=0.05$ and $C=0.5$. The shape parameter $c$ is choose $8.15 \times 10^{-2}$ and

Table 5: The comparison of the $L_{1}, L_{2}$ and RMS errors of our method with the results of $[7,22]$ at different times of experiment 4.

\begin{tabular}{|c|c|c|c|c|c|}
\hline Time & 1 & 2 & 3 & 4 & 5 \\
\hline \multicolumn{6}{|c|}{ IMQQI; N = 100} \\
\hline $\mathrm{L}_{1}$ & $4.9908 \mathrm{E}-07$ & $1.2551 \mathrm{E}-06$ & $1.7749 \mathrm{E}-06$ & $1.4689 \mathrm{E}-06$ & $7.3491 \mathrm{E}-07$ \\
\hline L2 & $1.3310 \mathrm{E}-06$ & $1.9435 \mathrm{E}-06$ & $3.3137 \mathrm{E}-06$ & $2.1256 \mathrm{E}-06$ & $2.2948 \mathrm{E}-06$ \\
\hline RMS & $1.3244 \mathrm{E}-07$ & $1.9339 \mathrm{E}-07$ & $3.2972 \mathrm{E}-07$ & $2.1150 \mathrm{E}-07$ & $2.9342 \mathrm{E}-07$ \\
\hline \multicolumn{6}{|c|}{ CBSM [22]; $\quad \mathrm{N}=200$} \\
\hline $\mathrm{L}_{1}$ & $3.5666 \mathrm{E}-06$ & $3.1949 \mathrm{E}-06$ & $3.9619 \mathrm{E}-06$ & $5.6889 \mathrm{E}-06$ & $6.3356 \mathrm{E}-06$ \\
\hline $\mathrm{L}_{2}$ & 2.5993E-05 & 2.2013E-05 & $2.3990 \mathrm{E}-05$ & 2.9542E-05 & $3.2638 \mathrm{E}-05$ \\
\hline RMS & $2.5930 \mathrm{E}-06$ & $2.2013 \mathrm{E}-06$ & $2.3909 \mathrm{E}-06$ & $2.9542 \mathrm{E}-06$ & $2.9092 \mathrm{E}-06$ \\
\hline TPSM [7]; & $\mathrm{N}=200$ & & & & \\
\hline $\mathrm{L}_{1}$ & $5.0705 \mathrm{E}-05$ & $5.0260 \mathrm{E}-04$ & $2.0612 \mathrm{E}-03$ & $6.5720 \mathrm{E}-03$ & $1.9067 \mathrm{E}-02$ \\
\hline $\mathrm{L}_{2}$ & 2.9474E-04 & $2.7082 \mathrm{E}-03$ & $9.7246 \mathrm{E}-03$ & $2.7881 \mathrm{E}-02$ & 7.7337E- 02 \\
\hline RMS & $2.0789 \mathrm{E}-05$ & $1.9102 \mathrm{E}-04$ & $6.8592 \mathrm{E}-04$ & $1.9666 \mathrm{E}-03$ & $5.4549 \mathrm{E}-03$ \\
\hline
\end{tabular}

$1.63 \times 10^{-2}$ for $N=100$ and $N=50$, respectively. Tables 3 and 4 indicate that the proposed method requires more less nodes to attain the accuracy of the CBSM [22] and TPSM [7].

Experiment 4. Consider the nonlinear Klein-Gordon equation (1) with $\mu=1$ and the nonlinear force $F(u)=u+u^{3}$ in interval $-1 \leqslant x \leqslant 1$. In this case, the constants $\alpha, \beta$ and $k$ are considered 1,1 and 3 , respectively. The initial conditions are given by

$u(x, 0)=x^{2} \cosh (x), \quad-1 \leqslant x \leqslant 1$

$u_{t}(x, 0)=x^{2} \sinh (x), \quad-1 \leqslant x \leqslant 1$.

The exact solution is given as

$u(x, t)=x^{2} \cosh (x+t)$.

The boundary function $g(x, t)$ can be extracted from the exact solution. Table 5 shows the $\mathrm{L}_{2}, \mathrm{~L}_{\infty}$ and RMS error norms in the solutions with $\Delta t=0.0001, c=8.15 \times 10^{-2}, N=100 \quad$ and $\gamma=\frac{1}{12}$. We compare our results with the results in [7, 22]. Also, the space-time graph of the estimated solution is drawn in Fig. 4. Table 5 shows that our scheme has better accuracy than CBSM [22] and TPSM [7]. 


\section{Conclusion}

In this paper, a numerical scheme based on high accuracy MQ quasi-interpolation scheme and IRBFN method has been applied to solve the nonlinear Klein-Gordon equation with quadratic and cubic nonlinearity. The numerical results which are given in the previous section demonstrate the good accuracy of the present scheme. Also, the Tables show that this scheme performs better than TPS method and requires more less nodes to attain accuracy. Moreover, we have used bigger time step $\Delta t$, in comparison with [13]. Therewith, we would like to emphasize that, the scheme introduced in this paper can be well studied for any other nonlinear PDEs.

\section{References}

[1] R.K. Beatson, M.J.D. Powell, "Univariate multiquadric approximation: quasi-interpolation to scattered data", Constr. Approx., 8 (3) (1992) 275-288.

[2] J. Biazar, M. Hosami, "Two effect approaches based on radial basis functions to nonlinear time-dependent partial differential equations", J. Math. Computer Sci., 9 (2014) 1-11.

[3] R.H. Chen, Z.M. Wu, "Solving hyperbolic conservation laws using multiquadric quasiinterpolation", Numer. Methods Partial Differential Equations, 22 (4) (2006) 776-796.

[4] R.H. Chen, Z.M. Wu, "Solving partial differential equation by using multiquadric quasiinterpolation", Appl. Math. Comput, 186 (2) (2007) 1502-1510.

[5] M. Dehghan, A. Shokri, "A numerical method for KdV equation using collation and radial basis functions", Nonlinear Dyn., 50 (2007) 111-120.

[6] M. Dehghan, A. Shokri, "A numerical method for solution of the two-dimensional sine-Gordon equation using the radial basis functions", Mathematics and Computers Simulation, 79 (2008) 700-715.

[7] M. Dehghan, A. Shokri, "Numerical solution of the nonlinear Klein-Gordon equation using radial basis functions", J. Comput. Appl. Math., 230 (2009) 400-410.

[8] Z.M. Wu, "Dynamically knots setting in meshless method for solving time dependent propagations equation", Comput. Methods Appl. Mech. Eng., 193 (12-14) (2004) 1221-1229.

[9] Z.M. Wu, "Dynamically knot and shape parameter setting for simulating shock wave by using multiquadric quasi-interpolation", Engineering Analysis with Boundary Elements, 29 (2005) 354-358.

[10] S.M. El-Sayed, "The decomposition method for studying the Klein-Gordon equation", Chaos Solitons Fractals, 18 (2003) 1025-1030.

[11] Y.C. Hon, X.Z. Mao, "An efficient numerical scheme for Burgers' equation", Appl. Math. Comput, 95 (1998) 37-50.

[12] Y.C. Hon, Z.M. Wu, "A quasi-interpolation method for solving stiff ordinary differential equations", Internat. J. Numer. Methods Eng., 48 (8) (2000) 1187-1197.

[13] E.J. Kansa, "Multiquadric-a scattered data approximation scheme with applications to computational fluid dynamics I", Comput. Math. Appl. 19 (1990) 127-145.

[14] E.J. Kansa, "Multiquadric-a scattered data approximation scheme with applications to computational fluid dynamics II", Comput. Math. Appl., 19 (1990) 147-161.

[15] D. Kaya, S.M. El-Sayed, "A numerical solution of the Klein-Gordon equation and convergence of the decomposition method", Appl. Math. Comput. , 156 (2004) 341-353.

[16] Z.W. Jiang, R.H. Wang, C.G. Zhu, M. Xu, "High accuracy multiquadric quasi-interpolation", Appl. Math. Modelling, 35 (2011) 2185-2195.

[17] Z.W. Jiang, R.H. Wang, "Numerical solution of one-dimensional sine-Gordon equation using high accuracy multiquadric quasi-interpolation", Appl. Math. comput. , 218 (2012) 7711-7716.

[18] S. Jimenez, L. Vazquez, "Analysis of four numerical schemes for a nonlinear Klein-Gordon equation”, Appl. Math. Comput. , 35 (1990) 61-94. 
[19] W.R. Madych, S.A. Nelson, "Multivariate interpolation and conditionally positive definite functions", Math. Comp., 54 (1990) 211-230.

[20] N. Mai-Duy, T. Tran-Cong, "Numerical solution of differential equations using multiquadric radial basis function networks", Neural Netw., 14 (2001) 185-199.

[21] J.K. Perring, T.H. Skyrme, “A model unified field equation”, Nucl. Phys. 31 (1962) 550-555.

[22] J. Rashidinia, M. Ghasemia, R. Jalilian, "Numerical solution of the nonlinear Klein-Gordon equation”, J. Comput. Appl. Math., 233 (2010) 1866-1878.

[23] M. Sarboland, A. Aminataei, "On the numerical solution of one-dimensional nonlinear nonhomogeneous Burgers equation", J. Appl. Math., 15 pages, doi:10.1155/2014/598432.

[24] M. Sarboland, A. Aminataei, "Improvement of the multiquadric quasi-interpolation $L_{\mathrm{W}_{2}}$ ", J. Math. Computer Sci., 11 (2014) 13-21.

[25] Sirendaoreji, "Auxiliary equation method and new solutions of Klein-Gordon equations", Chaos Solitons Fractals, 31(4) (2007) 943-950.

[26] Sirendaoreji, "A new auxiliary equation and exact travelling wave solutions of nonlinear equations", Phys. Lett. A, 356 (2) (2006) 124-130.

[27] C. Shu, L. Wu, "Integrated radial basis functions-based differential quadrature method and its performance", Int. J. Numer. Methods Fluids, 53 (2007) 969-984.

[28] A.M. Wazwaz, "The modified decomposition method for analytic treatment of differential equations", Appl. Math. Comput., 173 (2006) 165-176.

[29] Z.M. Wu, R. Schaback, "Shape preserving properties and convergence of univariate multiquadric quasi- interpolation, Acta.", Math. Appl. Sinica (English Ser.), 10 (4) (1994) 441 446.

[30] A.M. Wazwaz, "The tanh and the sine-cosine methods for compact and noncompact solutions of the nonlinear Klein-Gordon equation", Appl. Math.Comput., 167 (2005) 1179-1195.

[31] A.M. Wazwaz, "New travelling wave solutions to the Boussinesq and the Klein-Gordon equations", Communications in Nonlinear Science and Numerical Simulation, 13 (2008) 889901.

[32] M.L. Xiao, R.H. Wang, C.H. Zhu, "Applying multiquadric quasi-interpolation to solve KdV equation”, Mathematical Research Exposition, 31 (2011) 191-201. 